\title{
CHAOS OF A COUPLED LATTICE SYSTEM RELATED WITH BELUSOV-ZHABOTINSKII REACTION
}

\author{
JUAN LUIS GARCÍA GUIRAO ${ }^{1}$ AND MAREK LAMPART ${ }^{2}$
}

\begin{abstract}
In this paper we present a lattice dynamical system stated by K. Kaneko in [Phys. Rev. Lett., 65, 1391-1394, 1990] which is related to the Belusov-Zhabotinskii reaction. We prove that this CML (Coupled Map Lattice) system is chaotic in the sense of $\mathrm{Li}$ - Yorke and Devaney for zero coupling constant.
\end{abstract}

\section{INTRODUCTION}

Classical Discrete Dynamical Systems (DDS's), i.e., a couple composed by a space $X$ (usually compact and metric) and a continuous self-map $\psi$ on $X$, have been highly considered in the literature (see e.g., $[\mathrm{BC}]$ or $[\mathrm{De}]$ ) because are good examples of problems coming from the theory of Topological Dynamics and model many phenomena from biology, physics, chemistry, engineering and social sciences (see for example, [Da], [KO], [Pu] or [Po]). In most cases in the formulation of such models $\psi$ is a $C^{\infty}$, an analytical or a polynomial map.

Coming from chemical engineering applications, such a digital filtering, imaging and spatial vibrations of the elements which compose a given chemical product, a generalization of DDS's have recently appeared as an important subject for investigation, we mean the so called Lattice Dynamical Systems or 1d Spatiotemporal Discrete Systems. In the next section we provide all the definitions. To show the importance of these type of systems, see for instance [ChF].

To analyze when one of this type of systems have a complicated dynamics or not by the observation of one topological dynamics property

Key words and phrases. coupled map lattice, chaos in the sence of $\mathrm{Li}$ - Yorke, chaos in the sence of Devaney.

2000 Mathematics Subject Classification: 37L60, 37B99, 37B10. This research was supported in part by MEC (Ministerio de Educación y Ciencia, Spain) grants MTM2005-03860 and MTM2005-06098-C02-01; Fundación Séneca (Comunidad Autónoma de la Región de Murcia), grant 00684-FI-04, by Grant Agency of the Czech Republic grant 201/07/P032, Ministry of Education of the Czech Republic No. MSM6198910027 and VaV 1/09 of the Moravian-Silesian Region of the Czech Republic. 
is an open problem. The aim of the present paper is to show, by using the notion of chaos, and to characterize the dynamical complexity of coupled lattice systems stated by K. Kaneko in $[\mathrm{K}]$ (for more details see for references therein) which is related to the Belusov-Zhabotinskii reaction. We present some other problems for the future related with chemical applications.

to define chaos in the sence of Li - Yorke and Devaney (topological transitivity, locally eventually onto, periodically dense)

\section{Notation AND BAsic CONSTRUCTION}

The state space of LDS (Lattice Dynamical System) is the set

$$
\mathcal{X}=\left\{x \mid x=\left\{x_{i}\right\}, x_{i} \in \mathbb{R}^{d}, i \in \mathbb{Z}^{D},\left\|x_{i}\right\|<\infty\right\},
$$

where $d \geq 1$ is the dimension of the range space of the map of state $x_{i}, D \geq 1$ is the dimension of the lattice and the $l^{2}$ norm $\|x\|_{2}=$ $\left(\sum_{i \in \mathbb{Z}^{D}}\left|x_{i}\right|^{2}\right)^{1 / 2}$ is usually taken $\left(\left|x_{i}\right|\right.$ is the length of the vector $\left.x_{i}\right)$.

We deal with the following $1 \mathrm{~d}-L \mathrm{D}$ CML (Coupled Map Lattice) system which was stated by $\mathrm{K}$. Kaneko in $[\mathrm{K}]$ (for more details see for references therein) and it is related to the Belusov-Zhabotinskii reaction (see $[\mathrm{KO}]$ and for experimental study of chemical turbulence by this method [HGS], [HOY], [HHM]):

$$
x_{n}^{m+1}=(1-\epsilon) f\left(x_{n}^{m}\right)+\epsilon / 2\left[f\left(x_{n-1}^{m}\right)-f\left(x_{n+1}^{m}\right)\right],
$$

where $m$ is discrete time index, $n$ is lattice side index with system size $L$ (i.e. $n=1,2, \ldots L), \epsilon$ is coupling constant and $f(x)$ is the unimodal map on the unite closed interval $I=[0,1]$, i.e. $f(0)=f(1)=0$ and $f$ has unique critical point $c$ with $0<c<1$ such that $f(c)=1$. For simplicity we will deal with so called "tent map", defined by

$$
f(x)=\left\{\begin{array}{l}
2 x, \quad x \in[0,1 / 2) \\
2-2 x, \quad x \in[1 / 2,1]
\end{array}\right.
$$

In general, one for the following periodic boundary conditions of the system (1) is assumed:

(1) $x_{n}^{m}=x_{n+L}^{m}$,

(2) $x_{n}^{m}=x_{n}^{m+L}$,

(3) $x_{n}^{m}=x_{n+L}^{m+L}$,

standardly, the first case of the boundary conditions is used. 
The equation (1) was studied by many authors, mostly experimentally or semi-analytically then analytically. The first paper with analytic results is [ChL], where it was proved that this system is chaotic in the sense of $\mathrm{Li}$ - Yorke, we give alternative and easier proof in this paper.

We consider, as an example the 2-element one-way coupled logistic lattice (OCLL, see $[\mathrm{KW}]$ ) $H: I^{2} \rightarrow I^{2}$ written as

$$
\begin{aligned}
& x_{1}^{m+1}=(1-\epsilon) f\left(x_{1}^{m}\right)+\epsilon f\left(x_{2}^{m}\right), \\
& x_{2}^{m+1}=\epsilon f\left(x_{1}^{m}\right)+(1-\epsilon) f\left(x_{2}^{m}\right),
\end{aligned}
$$

where $f$ is the tent map.

\section{MAIN RESUlT}

The following two lemmas will be used for the proof of the main result. The proof of the first one is obvious (or, see e.g. $[\mathrm{DK}]$ ).

Lemma 1. Let $f: X \rightarrow X$ and $g: Y \rightarrow Y$ be periodically dense. Then the Cartesian product $f \times g: X \times Y \rightarrow X \times Y$ is also periodically dense.

Proposition 1. Let $f$ be the tent map defined by (2). Put $I_{k, l}=[(l-$ 1) $\left./ 2^{k}, l / 2^{k}\right]$ where $l=\left\{1,2,3, \ldots, 2^{k}\right\}$ and $k \in \mathbb{N}$. Then the restriction of $f^{k}$ to $I_{k, l}$ is linear homeomorphism onto $[0,1]$.

Let us note that the Cartesian product of two topologically transitive maps is not necessarily topologically transitive (see e.g. [DK] ). Hence, for the proof of Theorem 1 we need to prove:

Lemma 2. The system

$$
x_{n}^{m+1}=(1-\epsilon) f\left(x_{n}^{m}\right)+\epsilon / 2\left[f\left(x_{n-1}^{m}\right)-f\left(x_{n+1}^{m}\right)\right],
$$

is locally eventually onto for $\epsilon=0$.

Proof. Let $U$ be given open subset of $I^{L}$. Then the projection of $U$ to the $m$-th coordinate contains $U_{m}$ open connected subset of $I$, for each $m=1,2, \ldots L$. Then by Proposition 1 there is $k_{m}$ such that $f^{k_{m}}\left(U_{m}\right)=I$. If we put $K=\max \left\{k_{m} \mid m=1,2, \ldots L\right\}$ then the $K$-th iteration of $U$ by the system (1) equals to $I^{L}$.

Theorem 1. The system

$$
x_{n}^{m+1}=(1-\epsilon) f\left(x_{n}^{m}\right)+\epsilon / 2\left[f\left(x_{n-1}^{m}\right)-f\left(x_{n+1}^{m}\right)\right],
$$

is chaotic in the sense of Devaney for $\epsilon=0$.

Proof. The assertion follows by Lemma 1 and Lemma 2. 
The following Proposition is very powerful tool of symbolic dynamics $^{1}$ for observing nearly all dynamical properties.

Proposition 2. [GL] There is a subsystem of (1) which is conjugated ${ }^{2}$ to $\left(\Sigma_{2}^{L}, \sigma_{2}^{L}\right)$.

Theorem 2. The system

$$
x_{n}^{m+1}=(1-\epsilon) f\left(x_{n}^{m}\right)+\epsilon / 2\left[f\left(x_{n-1}^{m}\right)-f\left(x_{n+1}^{m}\right)\right],
$$

is chaotic in the sense of $L i-$ Yorke for $\epsilon=0$.

Proof. By Proposition 2 the system (1) has a subsystem conjugated to $\left(\Sigma_{2}^{L}, \sigma_{2}^{L}\right)$ which is $\mathrm{Li}-$ Yorke chaotic (see e.g. [BGKM]).

\section{Concluding Remarks}

There are many other notions of chaos, like distributional, omega or specification property. The system (1) fulfils all this chaotic behaviour by the same arguments as in the proof of the Theorem 2. But obviously this system is not minimal, where minimal means that there is no proper subset which is invariant, nonempty and closed.

For non-zero coupling constants the dynamical behaviour of the system (1) is more complicated. The first question is how the invariant subsets of phase space looks like? Secondly, what are the properties of $\omega$ limit sets? The answer for these questions will be nontrivial. Similar system was studied in [BGLL] and there was used the method of resultants to prove existence of periodic points of higher order. The same concept like in [BGLL] should be used.

\section{REFERENCES}

[AKM] R.L. Adler, A.G. Konheim and M.H. McAndrew, Topological entropy, Trans. Amer. Math. Soc., 114, 309-319, 1965.

[BC] L.S. Block and W.A. Coppel, Dynamics in One Dimension, Springer Monographs in Mathematics, Springer-Verlag, 1992.

[BCP] E. Bollt, N.J. Corron and S.D. Pethel, Symbolic dynamics of coupled map lattice, Phys. Rew. Lett., 96, 1-4, 2006.

[B] R. Bowen, Entropy for group endomorphisms and homogeneous spaces, Trans. Amer. Math. Soc., 153, 401-414, 1971.

[BGLL] F. Balibrea, J.L. Garca Guirao, M. Lampart and J. Llibre, Dynamics of a Lotka-Volterra map, Fund. Math. 191 3, 265-279, 2006.

\footnotetext{
${ }^{1}$ Here, $\sigma_{2}$ is the shift operator on the space of all two element sequences $\Sigma_{2}$.

${ }^{2}$ We say that two dynamical systems $(X, f)$ and $(Y, g)$ are topologically conjugated if there is a homeomorphism $h: X \rightarrow Y$ such that $h \circ f=g \circ h$, such homeomorphism is called conjugacy.
} 
[BGKM] F. Blanchard, E. Glasner, S. Kolyada and A. Maass, On Li-Yorke pairs, Journal fr die reine und angewandte Mathematik (Crelle's Journal), 547, $51-68,2002$.

[DK] N. Degirmenci and S. Kocak, Chaos in product maps, Turkish J. Math. to appear.

[GL] J.L. Garca Guirao and M. Lampart and J. Llibre, Positive entropy of a coupled lattice system related with Belusov-Zhabotinskii reaction, Journal of Math. Chem. - to appear.

[HHM] J.L. Hudson, M. Hart and D. Marinko, An experimental study of multiplex peak periodic and nonperiodic oscilations in the Belusov-Zhabotinskii reaction, J. Chem. Phys., 71, (1979), 1601-1606.

[HOY] K. Hirakawa, Y. Oono and H. Yamakazi, Experimental study on chemical turbulence. II, Jour. Phys. Soc. Jap., 46, (1979), 721-728.

[HGS] J.L. Hudson, K.R. Graziani and R.A. Schmitz, Experimental evidence of chaotic states in the Belusov-Zhabotinskii reaction, J. Chem. Phys., 67, (1977), 3040-3044.

[ChF] J.R. Chazottes and B. Fernndez, Dynamics of Coupled Map Lattices and of Related Spatially Extended Systems, Lecture Notes in Physics, 671, 2005.

[ChL] G. Chen and S. T. Liu, On spatial periodic orbits and spatial chaos, Int. J. of Bifur. Chaos, 13, 935-941, 2003.

[Da] R. A. Dana and L. Montrucchio, Dynamical Complexity in Duopoly Games, J. Econom. Theory, 40 (1986), 40-56

[De] R.L. Devaney, An Introduction to Chaotics Dynamical Systems, Benjamin/Cummings, Menlo Park, CA., 1986.

[Di] E.I. Dinaburg, A connection between various entropy characterizations of dynamical systems, Izv. Akad. Nauk SSSR Ser. Mat., 35 (1971), 324-366

[F] H. Furnsterbeg, Recurrence in Ergodic Theory and Combinational Number Theory. Princeton University Press. XI, Princeton, New Jersey, 1981.

[K] K. Kaneko, Globally Coupled Chaos Violates Law of Large Numbers, Phys. Rev. Lett., 65, 1391-1394, 1990.

[KO] M. Kohmoto and .Oono, Discrete model of Chemical Turbulence, Phys. Rev. Lett., 55, 2927 - 2931, 1985.

$[\mathrm{KW}]$ K. Kaneko and H.F. Willeboordse, Bifurcations and spatial chaos in an open flow model, Phys. Rew. Lett., 73, 533-536, 1994.

[LY] T. Y. Li and J. A. Yorke, Period three implies chaos. Amer. Math. Monthly, 82(10), 985992, 1975.

[Po] B. Van der Pool, Forced oscilations in a circuit with nonlinear resistence, London, Edinburgh and Dublin Phil. Mag, 3 (1927), 109-123.

$[\mathrm{Pu}]$ T. Puu, Chaos in Duopoly Pricing, Chaos, Solitions and Fractals, 1 (1991), 573-581.

[W] P. Walters, An introduction to ergodic theory. Springer, New York, 1982.

${ }^{1}$ Departamento de Matemática Aplicada y Estadística. Universidad

Politécnica de Cartagena, Hospital de Marina, 30203-Cartagena

(Región de Murcia), Spain. (CORresponding author)

E-mail address: juan.garcia@upct.es 
${ }^{2}$ Department of Applied Mathematics. VŠB - Technical University of Ostrava, 17. listopadu 15/2172, 70833 Ostrava, Czech Republic.

E-mail address: marek. lampart@vsb.cz 